\title{
Factors associated with high brachial-ankle pulse wave velocity in non-hypertensive and appropriately treated hypertensive patients with atherosclerotic risk factors
}

\author{
This article was published in the following Dove Press journal: \\ Vascular Health and Risk Management \\ 10 October 2017 \\ Number of times this article has been viewed
}

\author{
Dai Ato' \\ Toshitami Sawayama ${ }^{2}$ \\ 'Gakujutsu Shien (Academic Support) \\ Co., Ltd. Tokyo, ${ }^{2}$ Sawayama Clinic, \\ Okayama, Japan
}

\begin{abstract}
While pulse wave velocity (PWV) correlates with blood pressure (BP), its extent differs between patients, and some cases of high PWV in normotensives are present. Moreover, PWV frequently remains high in hypertensive patients despite adequate BP control. The factors associated with such phenomena are yet to be elucidated. Here, we investigated the factors associated with brachial-ankle PWV (baPWV) in 107 patients whose systolic BP was under $140 \mathrm{mmHg}$ at their latest baPWV measurement. There were 64 controlled hypertensives and 43 normotensives. Multivariate regression analysis identified age, hypertension, body mass index (BMI), systolic BP, and heart rate (HR) as independent factors for baPWV. Next, we divided the subjects into groups according to their age (in 5-year increments) and calculated the mean and standard deviation (SD) of the baPWV for each group. For each age group, we defined patients with a baPWV above the mean + SD baPWV for the group as the high-baPWV cohort. Multivariate logistic regression analysis revealed that BMI, hypertension, and smoking were independent determinants of a high-baPWV subject. This represents the first study to report the existence of the hypertensive state itself as one of the independent predictors of high baPWV in normotensive and well-treated hypertensive patients. This finding implies that the hypertensive state itself possibly worsens arterial stiffness independently from aging in spite of adequate BP maintenance. To prevent the early progression of arterial stiffness, the application of an appropriate intervention during the early stages of hypertension is important and the continuation of an appropriate BP treatment is suggested.
\end{abstract}

Keywords: arterial stiffness, brachial-ankle pulse wave velocity, heart failure, hypertension, peripheral arterial disease

\section{Introduction}

Brachial-ankle pulse wave velocity (baPWV) is an index of arterial stiffness that shows prognostic predictability independently from conventional atherosclerotic risk factors. ${ }^{1,2}$ In Western countries, evidence is mounting to indicate that aortic PWV represents a useful prognostic marker for arterial stiffness. ${ }^{3,4}$ The baPWV shows a strong correlation with aortic $\mathrm{PWV}^{5-7}$ and a significant number of studies have demonstrated the prognostic significance of baPWV over recent years. ${ }^{8-10}$ Hypertension is the strongest risk factor for cardiovascular diseases, and adequate blood pressure (BP) control is essential in the prevention of such diseases. ${ }^{2}$ PWV generally depends upon BP, but its extent is known to differ between patients. Depending on certain conditions, PWV sometimes displays independence from changes in BP. ${ }^{5}$ Furthermore, even when orrespondence: Dai Ato

Gakujutsu Shien (Academic Support) Co., Ltd., 2-I-I 7 Nihonbashi, Chuo-ku, Tokyo, 103-0027, Japan

Tel +8I 368696438

Email ato.dail@wind.ocn.ne.jp 
BP is well controlled, there are cases of reduced PWV and frequent cases of PWV remaining high without improvement (Figure 1A and B). It is possible that this condition indicates a residual risk. ${ }^{8,9}$ However, factors associated with these phenomena have not been fully elucidated. Therefore, in the present study, we investigated the potential association between baPWV and a variety of factors in outpatients attending our clinic, both with and without hypertension, and in whom systolic BP was controlled to less than $140 \mathrm{mmHg}$.

\section{Methods}

\section{Subjects}

We recruited outpatients attending the Sawayama Clinic (Kurashiki city, Okayama, Japan) who had undergone simultaneous baPWV/ankle--brachial index (ABI) measurements at least three times before the end of October 2014 and who had bilateral baPWV values at the latest opportunity. Figure 2 depicts the patient selection process. The condition of baPWV/ABI measured at least three times was stipulated to
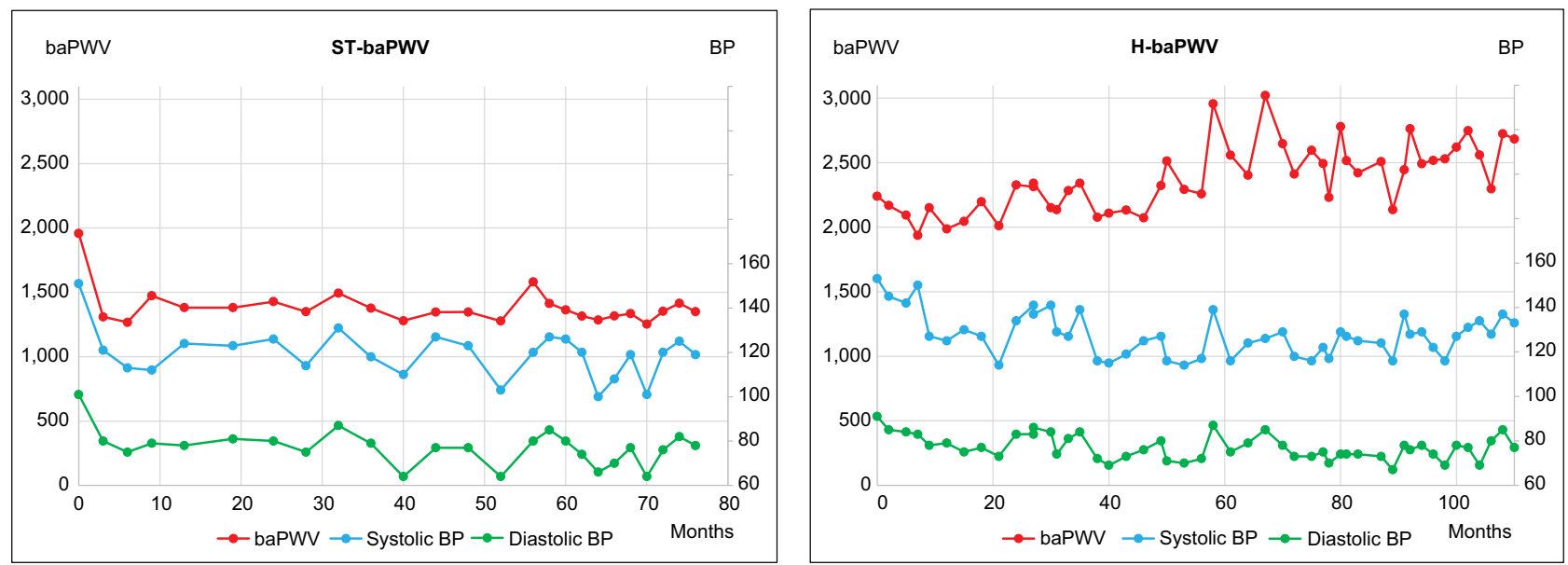

Figure I Trend graphs of two typical blood pressure-controlled patients with or without improved baPWV.

Notes: (A) Born in May, 1942, male, hypertension. Minor old myocardial infarction was seen during first visit, April 2010;. baPWV was relatively high at 2,000 cm/s but improved with BP reduction and was kept stable at approximately $1,400 \mathrm{~cm} / \mathrm{s}$ for several years. (B) Born in March $194 \mathrm{I}$, male, hypertension, diabetes, smoking, minor cardiomegaly. Antihypertensive treatment was started in May 2007. Despite adequate blood pressure control, baPWV did not improve but increased 5 years after the initiation of antihypertensives and remained at approximately $2,500 \mathrm{~cm} / \mathrm{s}$ with perturbation.

Abbreviations: baPWV, brachial-ankle pulse wave velocity; BP, blood pressure; $\mathrm{H}$, high; ST, standard.

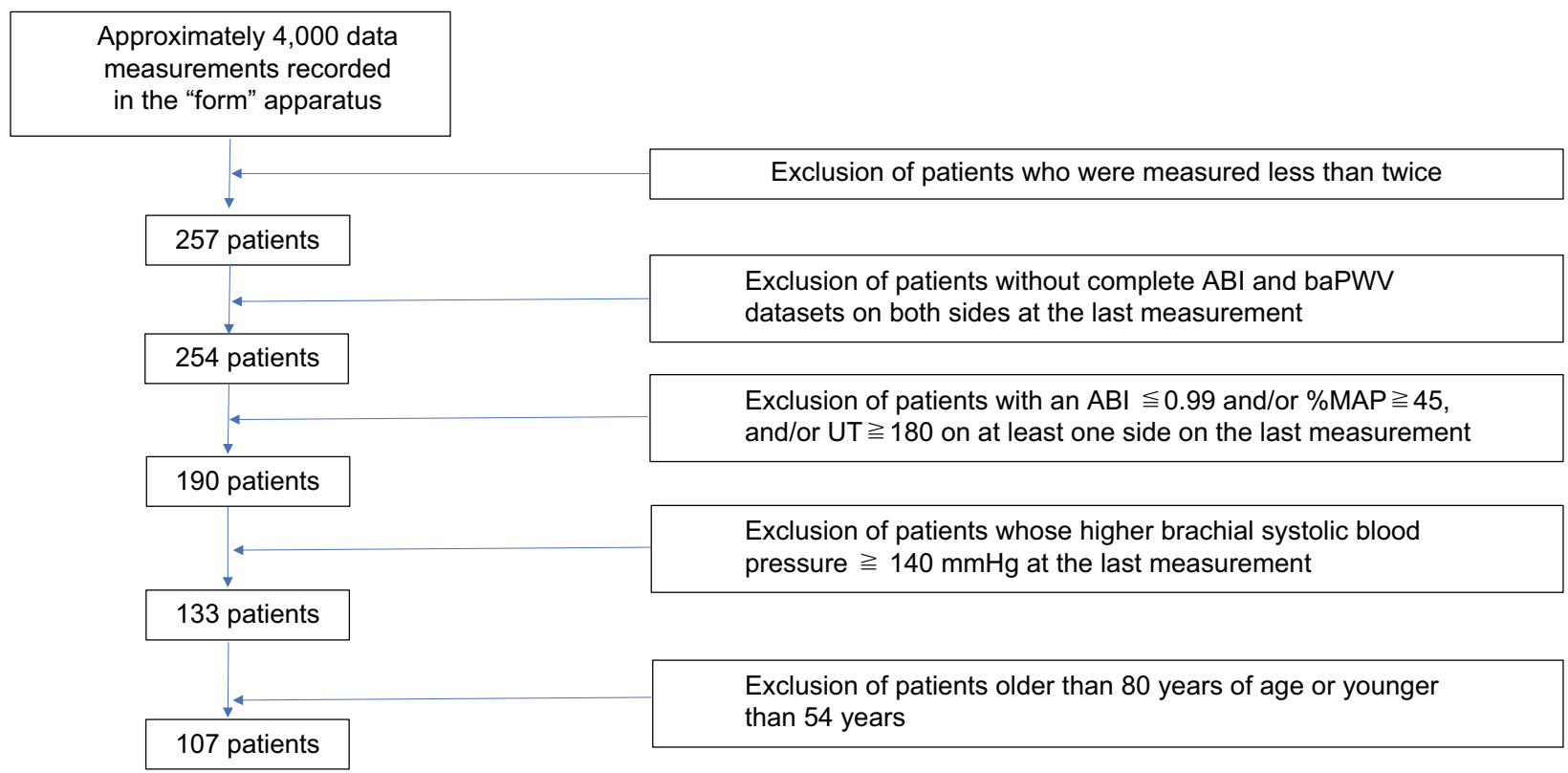

Figure 2 Flow chart showing the patient selection procedure.

Abbreviations: $\mathrm{ABI}$, ankle-brachial index; baPWV, brachial-ankle pulse wave velocity; \%MAP, percent mean arterial pressure; UT, upstroke time. 
ensure that we selected patients whose BP was continuously controlled over a definite period through hospital attendance. We did not include patients if data arising from unilateral baPWV, and/or ABI, were lacking in the latest opportunity. Moreover, we excluded patients who had an $\mathrm{ABI} \leq 0.99$, a percent mean arterial pressure (\%MAP) of the ankle pulse waveform $\geq 45 \%$, or an upstroke time (UT) $\geq 180 \mathrm{~ms}$, at least unilaterally in the last examination; this was done to exclude possible cases of arteriosclerosis obliterans (ASO) and the underestimation of baPWV. ${ }^{1,11-13}$ Furthermore, we excluded data from patients in whom systolic BP was $>140 \mathrm{mmHg}$ in their most recent test, as these patients could not be BP controls. ${ }^{2}$ We excluded patients $<54$ or $>80$ of age due to the small numbers of such patients ( $n=14$ and $n=12$, respectively). Finally, 107 patients, between 55 and 79 years of age, were included for analysis. Although four of these patients had a diastolic BP $>90 \mathrm{mmHg}$, we did not exclude them. This was because diastolic BP showed no significant correlation with baPWV in this cohort, and we believed that these patients would have no significant influence on our statistical analysis. The data were obtained from the records of the Sawayama Clinic and were fully anonymized before analysis by the authors. The Sawayama Clinic permitted waiver of informed consent from subjects because of the retrospective study design and the fact that de-identified data were being accessed. The authors agreed with the study protocol, and the study was ethically conducted in accordance with the principles of the Declaration of Helsinki.

\section{Measurements and variables}

All variables related to $\mathrm{BP}$ and pulse wave examination were measured using a pulse waveform analyzer BP-203RPE II (form PWV/ABI, Nippon Colin, Komaki, Aichi, Japan). Details of the function and utility of this device have been described previously. ${ }^{1,5}$ Detailed explanations pertaining to \%MAP and UT, derived from ankle pulse waveforms, have also been provided previously. ${ }^{1,11,13}$ This device was also used to measure HR. For brachial BP, higher systolic BP and diastolic BP were used. Surrogate markers for arterial stiffness and lower limb atherosclerosis, higher baPWV, and lower ABI were used. ${ }^{1}$ For additional surrogate markers of lower limb atherosclerosis, higher UT and \%MAP were also selected. Moreover, this apparatus provided indices related to left ventricle systolic time intervals (STI), such as ejection time (ET), pre-ejection period (PEP), and ET/PEP. ${ }^{14,15}$ In five patients, at least one of the STI indices was not measured; thus, in terms of STI indices, data were collated from the remaining 102 patients. Most of the measurements from this device were conducted in the morning (106 out of 107). BMI was calculated as weight divided by height squared $\left(\mathrm{kg} / \mathrm{m}^{2}\right)$.

\section{Definition of atherosclerotic risk factors}

Hypertension was said to be present when BP recorded was $>140 / 90 \mathrm{mmHg}$ and/or when a patient was treated with antihypertensive medications. Diabetes and dyslipidemia were defined according to the current Japanese guidelines, ${ }^{16,17}$ including on the use of medications. Smoking was defined as having a regular smoking habit at the point when the last baPWV measurement was taken.

\section{Statistical analysis}

Continuous variables are expressed as means \pm standard deviations (SDs), while nominal variables are expressed as percentage ratios $(\%)$. We compared characteristics between sexes and between patients with and without risk factors (hypertension, diabetes, dyslipidemia, or smoking), coronary arterial disease (CAD), and cerebrovascular disease (CVD). We identified indices correlating with baPWV using the Pearson correlation method and conducted multivariate regression analysis of baPWV as a dependent variable. Next, we divided patients into five groups according to their age, 55-59 years $(n=16), 60-64$ years $(n=31), 65-69$ years $(n=25), 70-74$ years $(n=20)$, and 75-79 years $(n=15)$. We then calculated the mean baPWV \pm SD for each age group. We defined those with a baPWV $\leq$ mean $+\mathrm{SD}$ in each group as the standard baPWV (ST-baPWV, $\mathrm{n}=90$ ), and those with a baPWV $>$ mean + SD in each group as high baPWV (H-baPWV, $n=17)$. Then, we undertook multivariate logistic regression analysis to identify independent determinants of H-baPWV. We conducted the Mann-Whitney-Wilcoxon test for continuous variables and the chi-square test for nominal variables to compare the two groups using Microsoft Excel 2016. Two multivariate analyses were also performed using EZR (EZR on R commander Version 1.33, September 1, 2016). ${ }^{18}$ Multivariate analysis was based on a stepwise forward-backward procedure to determine independent factors. All the conventional risk factors (hypertension, diabetes, dyslipidemia, and smoking), and any variables for which probability $(p)$ was identified by univariate analysis as $<0.1$ were also included in the model, except for indices of pulse waveforms (UT, \%MAP, ET, and PEP). Statistical significance was set at $p<0.05$ (two tailed).

\section{Results}

Table 1 shows the characteristics of all 107 patients, classified by sex, and highlighting those with ST-baPWV and those with H-baPWV. Taking all patients into account, mean 
Table I Patient characteristics according to sex and baPWV category

\begin{tabular}{|c|c|c|c|c|c|c|c|}
\hline & $\begin{array}{l}\text { All } \\
(N=107)\end{array}$ & $\begin{array}{l}\text { Male } \\
(\mathrm{N}=67)\end{array}$ & $\begin{array}{l}\text { Female } \\
(\mathrm{N}=40)\end{array}$ & $p^{*}$ & $\begin{array}{l}\text { ST-baPWV } \\
(\mathrm{N}=90)\end{array}$ & $\begin{array}{l}\text { H-baPWV } \\
(\mathrm{N}=17)\end{array}$ & $p^{* *}$ \\
\hline Age (years) & $66 \pm 6$ & $66 \pm 7$ & $66 \pm 6$ & 0.94 & $66 \pm 6$ & $67 \pm 7$ & 0.64 \\
\hline Height (cm) & $164 \pm 8$ & $168 \pm 5$ & $158 \pm 6$ & $<0.0000$ I & $164 \pm 8$ & $164 \pm 8$ & 0.88 \\
\hline BMI $\left(K g / m^{2}\right)$ & $22.8 \pm 2.9$ & $23.2 \pm 2.6$ & $22.0 \pm 3.1$ & $<0.05$ & $23.1 \pm 2.8$ & $20.8 \pm 2.5$ & $<0.002$ \\
\hline Systolic BP (mmHg) & $124 \pm 10$ & $122 \pm 10$ & $126 \pm 9$ & $<0.03$ & $123 \pm 9$ & $126 \pm 9$ & 0.17 \\
\hline Diastolic BP (mmHg) & $76 \pm 8$ & $76 \pm 9$ & $77 \pm 9$ & 0.62 & $78 \pm 7$ & $76 \pm 8$ & 0.23 \\
\hline baPWV (cm/s) & $1644 \pm 302$ & $1624 \pm 286$ & $1678 \pm 329$ & 0.4 & $1556 \pm 206$ & $2112 \pm 301$ & $<0.0000$ I \\
\hline ABI & I. $14 \pm 0.07$ & $1.15 \pm 0.08$ & $1.13 \pm 0.05$ & 0.14 & $1.14 \pm 0.07$ & $1.16 \pm 0.07$ & 0.22 \\
\hline \%MAP & $38 \pm 3$ & $38 \pm 3$ & $40 \pm 2.8$ & $<0.0001$ & $38 \pm 3$ & $39 \pm 3$ & 0.56 \\
\hline UT (msec) & $148 \pm 16$ & $146 \pm 16$ & $150 \pm 15$ & 0.16 & $148 \pm 15$ & $146 \pm 20$ & 0.67 \\
\hline HR (bpm) & $64 \pm 10$ & $63 \pm 11$ & $65 \pm 9$ & 0.41 & $63 \pm 10$ & $68 \pm 9$ & $<0.04$ \\
\hline ET (msec) & $289 \pm 25$ & $286 \pm 25$ & $295 \pm 23$ & $<0.05$ & $292 \pm 23$ & $275 \pm 28$ & $<0.04$ \\
\hline PEP (msec) & $106 \pm 14$ & $107 \pm 15$ & $105 \pm 12$ & 0.65 & $106 \pm 14$ & $105 \pm 12$ & 0.75 \\
\hline ET/PEP & $2.78 \pm 0.45$ & $2.74 \pm 0.48$ & $2.82 \pm 0.41$ & 0.23 & $2.80 \pm 0.44$ & $2.65 \pm 0.46$ & 0.25 \\
\hline Sex (male, \%) & $63 \%$ & - & - & - & $62 \%$ & $65 \%$ & 0.91 \\
\hline H-baPWV (\%) & $16 \%$ & $16 \%$ & $15 \%$ & 0.84 & - & - & - \\
\hline Diabetes (\%) & $16 \%$ & $25 \%$ & $0 \%$ & $<0.002$ & $16 \%$ & $18 \%$ & 0.84 \\
\hline Hypertension (\%) & $60 \%$ & $63 \%$ & $55 \%$ & 0.62 & $56 \%$ & $82 \%$ & 0.19 \\
\hline Dyslipidemia (\%) & $45 \%$ & $45 \%$ & $45 \%$ & 0.99 & $46 \%$ & $41 \%$ & 0.8 \\
\hline Smoking (\%) & $37 \%$ & $58 \%$ & $3 \%$ & $<0.0000$ I & $34 \%$ & $53 \%$ & 0.25 \\
\hline CAD (\%) & $13 \%$ & $18 \%$ & $5 \%$ & 0.07 & $13 \%$ & $12 \%$ & 0.87 \\
\hline CVD (\%) & $3 \%$ & $3 \%$ & $3 \%$ & 0.88 & $2 \%$ & $6 \%$ & 0.41 \\
\hline OHD (\%) & $7 \%$ & $3 \%$ & $13 \%$ & 0.06 & $6 \%$ & $12 \%$ & 0.36 \\
\hline
\end{tabular}

Notes: $p^{*}$, comparison between males and females; $p^{* *}$, comparison between the ST-baPWV group and the H-baPWV group. Data shown as mean \pm SD, percentage, or $p$-value.

Abbreviations: $\mathrm{ABI}$, ankle-brachial index; $\mathrm{BMI}$, body mass index; $\mathrm{BP}$, blood pressure; baPWV, brachial-ankle pulse wave velocity; CAD, coronary artery disease; CVD, cerebrovascular disease; ET, ejection time; H, high; HR, heart rate; \%MAP, percent mean arterial pressure; OHD, other heart disease; PEP, pre-ejection period; ST, standard; UT, upstroke time.

age was $66 \pm 6$ years, mean height was $164 \pm 8 \mathrm{~cm}$, mean BMI was $22.8 \pm 2.9$, mean systolic BP was $124 \pm 10 \mathrm{mmHg}$, mean baPWV was $1644 \pm 302 \mathrm{~cm} / \mathrm{s}$, and mean ABI was $1.14 \pm 0.07$. Within our patient cohort, the proportion of those with hypertension was $60 \%(n=64)$, diabetes $16 \%(n=17)$, dyslipidemia $45 \%(n=48)$, smoking 37\% $(n=40)$, CAD 13\% $(n=14)$, and CVD 3\% (n=3). Other heart diseases (OHD), rather than CAD, were identified in seven patients, including atrial fibrillation $(n=2)$, ventricular tachycardia $(n=1)$, supraventricular arrhythmia $(n=1)$, sick sinus syndrome $(n=1)$, hypertrophic cardiomyopathy $(n=1)$, and mitral valve prolapse $(n=1)$. Comparing sexes, males were significantly taller $(168 \pm 5 \mathrm{~cm}$ vs $158 \pm 6 \mathrm{~cm}$, $p<0.00001)$ and had a significantly higher BMI $(23.2 \pm 2.6 \mathrm{~kg} /$ $\mathrm{m}^{2}$ vs $\left.22.0 \pm 3.1 \mathrm{~kg} / \mathrm{m}^{2}, p<0.05\right)$. Systolic BP was significantly higher in females $(122 \pm 10 \mathrm{mmHg}$ vs $126 \pm 9 \mathrm{mmHg}, p<0.03)$, but there was no significant difference between sexes in terms of baPWV. The 17 patients with diabetes were male only $(25 \%$ male vs $0 \%$ female, $p<0.002)$. Smokers were mostly males (39 out of 40 smokers); the smoking ratio was also higher in males $(58 \%$ vs $3 \%, p<0.00001)$. The incidence of CAD among males was higher than among females (18\% vs $5 \%$, $p=0.07$ ), whereas OHD was more common in females than among males (13\% vs 3\%, $p=0.06)$.
Table 1 shows a comparison between patients with STbaPWV (n=90) and H-baPWV (n=17). Age and systolic BP were not significantly different between those with

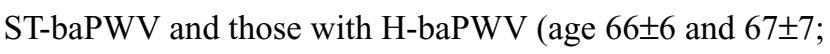
systolic BP: $123 \pm 9 \mathrm{mmHg}$ and $126 \pm 9 \mathrm{mmHg}$, respectively). The baPWV was significantly higher in the H-baPWV group $(1,556 \pm 206 \mathrm{~cm} / \mathrm{s}$ in ST-baPWV patients and 2,112 $\pm 301 \mathrm{~cm} / \mathrm{s}$ in $\mathrm{H}-\mathrm{baPWV}$ patients, $p<0.00001)$. Furthermore, baPWV increased with age in both the ST-baPWV and H-baPWV groups although there was no significant difference in systolic BP among different generations (Figure 3). Ratios of hypertension and smoking were not significantly different when compared between these two groups (hypertension: 56\% and $82 \%$, smoking: $34 \%$ and $53 \%$, respectively). BMI was significantly lower in the H-baPWV group $\left(23.1 \pm 2.8 \mathrm{~kg} / \mathrm{m}^{2}\right.$ and $20.8 \pm 2.5 \mathrm{~kg} / \mathrm{m}^{2}, p<0.002$ ), whereas HR was significantly higher in the H-baPWV group $(63 \pm 10 \mathrm{bpm}$ and $68 \pm 9 \mathrm{bpm}$, $p<0.04)$.

Table 2 depicts our univariate analysis of factors and baPWV. There were significant correlations between baPWV and age $(\mathrm{R}=0.49, p<0.00001)$, $\mathrm{BMI}(\mathrm{R}=-0.24, p<0.02)$, systolic $\mathrm{BP}(\mathrm{R}=0.26, p<0.01), \mathrm{HR}(\mathrm{R}=0.27, p<0.01)$, and hypertension $(\mathrm{R}=0.32, p<0.001)$. However, baPWV did 


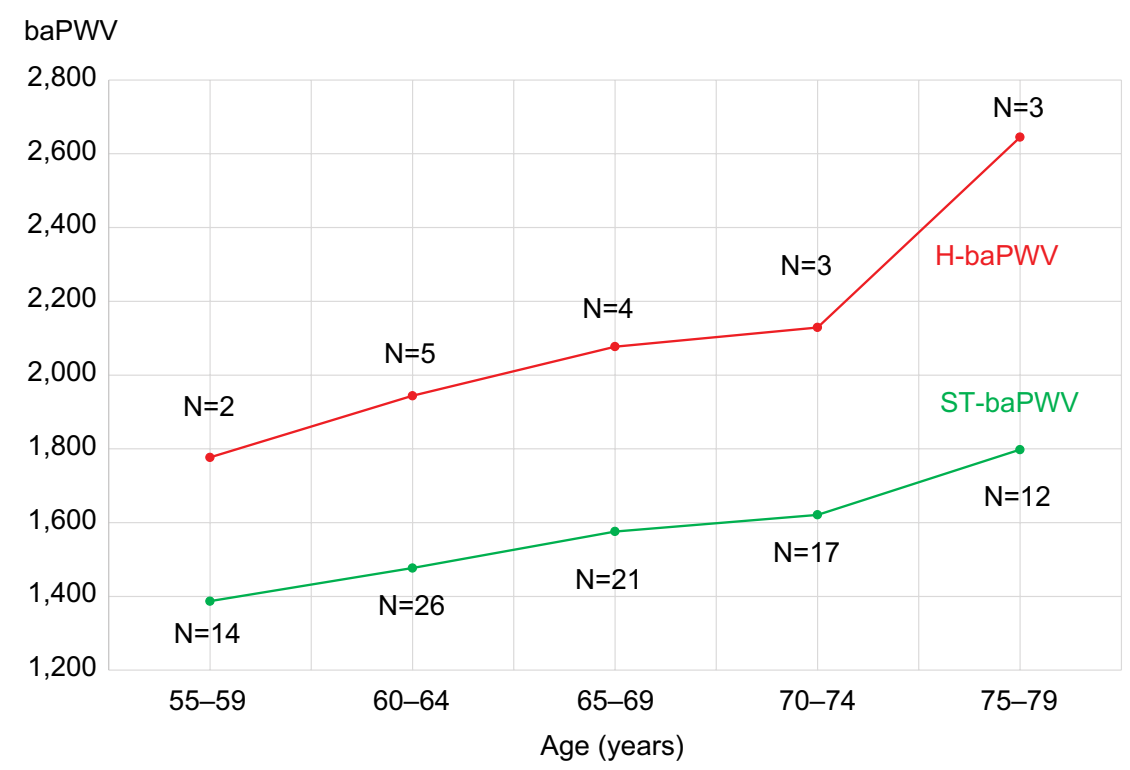

Figure 3 The baPWV according to age and category.

Abbreviations: baPWV, brachial-ankle pulse wave velocity; $\mathrm{H}$, high; ST, standard.

Table 2 Univariate and multivariate regression analysis of factors and baPWV as a continuous variable

\begin{tabular}{|c|c|c|c|c|c|c|}
\hline \multicolumn{3}{|l|}{ Univariate analysis } & \multicolumn{4}{|c|}{ Multivariate analysis } \\
\hline & $\boldsymbol{R}$ & $p$ & B & $\beta$ & $t$ & $p$ \\
\hline Age (years) & 0.49 & $<0.00001$ & 19.84 & 0.43 & 6.00 & $<0.00001$ \\
\hline Height (cm) & -0.18 & $<0.07$ & & & & \\
\hline BMI $\left(\mathbf{k g} / \mathbf{m}^{2}\right)$ & -0.24 & $<0.02$ & -30.12 & -0.28 & 3.93 & $<0.001$ \\
\hline Sex $($ male $=I)$ & -0.09 & 0.38 & & & & \\
\hline Systolic BP (mmHg) & 0.26 & $<0.01$ & 8.01 & 0.25 & 3.52 & $<0.001$ \\
\hline ABI & 0.13 & 0.19 & & & & \\
\hline HR (bpm) & 0.27 & $<0.01$ & 6.83 & 0.23 & 3.34 & $<0.01$ \\
\hline Diabetes (yes=I) & 0.01 & 0.93 & & & & \\
\hline Hypertension (yes $=I$ ) & 0.32 & $<0.001$ & 184.08 & 0.61 & 4.13 & $<0.0001$ \\
\hline Dyslipidemia (yes=I) & 0.03 & 0.8 & & & & \\
\hline Smoking $($ yes $=1)$ & 0.05 & 0.63 & & & & \\
\hline CAD $($ yes $=I)$ & -0.02 & 0.87 & & & & \\
\hline CVD (yes=I) & 0.05 & 0.58 & & & & \\
\hline OHD (yes=I) & -0.05 & 0.59 & & & & \\
\hline
\end{tabular}

Note: $R$-squared, 0.50 ; adjusted $R$-squared: 0.48 . Multivariate model included variables as follows: age, height, BMI, sex, systolic BP, HR, diabetes, hypertension, dyslipidemia, and smoking.

Abbreviations: $\mathrm{ABI}$, ankle-brachial index; $\mathrm{B}$, regression coefficient; BMI, body mass index; BP, blood pressure; CAD, coronary artery disease; CVD, cerebrovascular disease; $\mathrm{HR}$, heart rate; OHD, other heart disease; $R$, Pearson correlation coefficient; $\beta$, standardized regression coefficient.

not correlate with diabetes, dyslipidemia, smoking, CAD, CVD, or OHD. Table 2 also depicts the results arising from our multivariate regression analysis using baPWV as a continuous variable. Age $(\beta=0.43, p<0.00001)$, hypertension $(\beta=0.61, p<0.0001)$, systolic $\mathrm{BP}(\beta=0.25, p<0.001)$, BMI $(\beta=-0.28, p<0.001)$, and HR $(\beta=0.23, p<0.01)$ were identified as independent determinants of baPWV. Of these factors, patient age was the strongest determinant $(t=6.00)$; among the other factors, hypertension had the most powerful influence $(\beta=0.61, t=4.13)$.

Finally, Table 3 presents the results arising from our multivariate logistic regression analysis for $\mathrm{H}-\mathrm{baPWV}$ as a
Table 3 Multivariate logistic regression analysis of $\mathrm{H}$-baPWV determinants

\begin{tabular}{lll}
\hline & OR $(95 \% \mathrm{CI})$ & $\boldsymbol{p}$ \\
\hline BMI (SD) & $0.57(0.42-0.78)$ & $<0.00 \mathrm{I}$ \\
Hypertension & $6.4 \mathrm{I}(1.46-28.1)$ & $<0.02$ \\
Smoking & $4.62(1.23-17.3)$ & $<0.03$ \\
\hline
\end{tabular}

Note: Multivariate model included variables as follows: BMI, systolic blood pressure, $\mathrm{HR}$, diabetes, hypertension, dyslipidemia, and smoking.

Abbreviations: baPWV, brachial-ankle pulse wave velocity; BMI, body mass index; $\mathrm{Cl}$, confidence interval; $\mathrm{H}$, high $\mathrm{HR}$, heart rate; $\mathrm{OR}$, odds ratio; $\mathrm{SD}$, standard deviation.

dependent variable. Independent determinants of H-baPWV were BMI (odds ratio [OR] 0.57, 95\% confidence interval [CI] 0.42-0.78, $p<0.001$ ), hypertension (OR 6.41, 95\% 
CI $1.46-28.1, p<0.02$ ), and smoking (OR 4.62, 95\% CI $1.23-17.3, p<0.03)$. In addition, HR showed a tendency to represent an independent determinant, although this was not statistically significant (OR 1.06, 95\% CI $0.99-1.13$, $p=0.055$ ).

\section{Discussion}

To the best of our knowledge, this is the first study to demonstrate that the hypertensive state itself is a determinant of high baPWV in patients with adequately controlled BP. A phenomenon that correlates baPWV with systolic BP is consistent with its properties despite the fact that the systolic BP of our study subjects was $<140 \mathrm{mmHg}$. Nevertheless, multivariate regression analysis showed that systolic BP influenced only approximately $70 \mathrm{~cm} / \mathrm{s}$ of baPWV for every SD increase in systolic BP. On the other hand, the hypertensive state itself emerged as an independent determinant that could increase baPWV to $184 \mathrm{~cm} / \mathrm{s}$ despite the adequate achievement of an antihypertensive condition. This influence is the equivalent of aging by 9 years $(184.1 \div 19.8=9.3)$. Furthermore, those in a hypertensive state were predicted to develop H-baPWV by an OR of six. These results imply that the hypertensive state itself is a candidate predictor for H-baPWV. In contrast, there was no difference in baPWV for those with or without diabetes and dyslipidemia. These results, particularly the fact that baPWV was not associated with diabetes mellitus, are contradictory to the results of a recent Mendelian randomization study, which indicated the causal role of diabetes on baPWV. ${ }^{19}$ For this reason, we consider that the rate of diabetes was relatively low in our subjects (16\%). Moreover, our institute is a cardiology clinic that focuses particularly upon primary prevention. Consequently, there may be differences in terms of our patient characteristics as compared with those attending other institutes or departments, such as general medicine, diabetic clinics, or a foundation hospital. Our finding that baPWV had no association with dyslipidemia is plausible for several reasons, including the findings of a recent Mendelian randomization study that has evaluated triglyceride as a causative factor. ${ }^{20-22}$ Dyslipidemia is a factor that mainly affects the formation of intimal hyperplasia and plaque formation. On the other hand, baPWV is a factor that mainly reflects the stiffness or function of an aortic wall..$^{5-7}$ Furthermore, statins are mainly used to treat low-density lipoprotein cholesterol, and several studies have demonstrated differences in baPWV improvement, which relate to different types of statins. This suggests variance in pleiotropy, including antioxidative effects. ${ }^{23-25}$ For these reasons, dyslipidemia did not associate strongly with baPWV.
BMI was identified as a negative independent determinant of baPWV as a continuous variable and H-baPWV. A similar report has been published for a health check-up population in which the reference value of baPWV in each gender was calculated. Furthermore, it showed that BMI was independently associated with baPWV in a negative manner. ${ }^{21}$ Generally, lean individuals possibly have lesser volume of circulating blood and have arteries of smaller caliber (higher PWV), especially if BPs are similar. Our result would not contradict this logic. However, the influence of BMI in our study was approximately threefold higher than that of the health check-up population ( $\beta=-0.09$ in males, $\beta=-0.08$ in females). ${ }^{21}$ There are several different factors between our study subjects and the health check-up population. Those are mean age ( $\sim 20$ years), mean baPWV $(\sim 400 \mathrm{~cm} / \mathrm{s})$, and independent determinants identified by multivariate analysis. ${ }^{21}$ Mechanisms related to body composition such as sarcopenia could also have influenced baPWV. Ochi et al investigated the relationship between baPWV and sarcopenia in a cohort of middle-aged and elderly patients. They showed that the ratio of thigh muscle cross-sectional area to body weight correlated in a negative independent manner with baPWV. ${ }^{26}$ There have also been continuous reports of the "obesity paradox" over recent years. ${ }^{27-30}$ Especially, we noted striking evidence which showed simply, "the leaner they are, the earlier they decease," in a Japanese elderly cohort (aged 65 79) of the Japan Collaborative Cohort study. ${ }^{27}$ A synchronized phenomenon of 1) weight reduction mainly due to muscle mass reduction, 2) aortic stiffness progression, and 3) the trajectory of prognostic disadvantage from the aspect of baPWV in middle-aged and elderly patients may be consistent. Another study reported regional differences in PWVs in terms of correlation with BMI. ${ }^{31}$ Further studies are now needed to clarify these mechanisms.

In the present study, smoking emerged as an independent factor for H-baPWV although baPWV did not differ significantly between those with or without smoking. Furthermore, some studies have shown inconsistent results in terms of the influence of smoking upon baPWV. ${ }^{21,32-37}$ According to these reports, smoking acted acutely to increase baPWV as a whole. Nevertheless, in terms of long-term effects, smoking might have possible interactions with a variety of other factors, including exercise habits, total amount of smoking, smoking duration, and genetic factors. ${ }^{36,37}$ These interactions may, thus, exert influences upon baPWV.

We found, moreover, that HR was an independent factor for baPWV as a continuous variable and tended to be a determinant of $\mathrm{H}-\mathrm{baPWV}$. Compared to subjects in previous 
studies, who also showed an association between HR and baPWV, ${ }^{38,39}$ our subjects were older. This phenomenon might possibly be related to sympathetic nerve activity. ${ }^{40-42}$

We first demonstrated that the hypertensive state itself represents an independent factor for both baPWV and H-baPWV, especially among patients without hypertension and patients with appropriately treated hypertension. This result may represent a potential countermeasure for a future "heart failure (HF) pandemic." ${ }^{43,44}$ The baPWV has already been proved to be a predictor of all-cause mortality and cardiovascular events, even after adjustment for conventional atherosclerotic risk factors. ${ }^{8-10}$ Moreover, using baPWV with a cutoff of $1,750 \mathrm{~cm} / \mathrm{s}$, recurrent $\mathrm{HF}$ in those with normal BP and the onset of HF can be efficiently predicted. ${ }^{45,46}$ Knowledge of baPWV also enhances our ability to diagnose heart failure with preserved ejection fraction (HFpEF) in patients with acute dyspnea. ${ }^{47}$ Cong et al reported that the diagnostic accuracy for HFpEF significantly improved after adding baPWV to the diagnostic criteria of the 2007 ESC consensus statement (net reclassification improvement $0.127, p=0.02$ ). ${ }^{47}$ Collectively, these data suggest that baPWV has the diagnostic ability to predict patients with a high risk of $\mathrm{HF}$ and has adjunctive value to diagnose HFpEF. Moreover, this examination can simultaneously measure indices for left ventricle (LV) function; ET/PEP can accurately predict a reduced ejection fraction and LV dyssynchrony. ${ }^{15,48}$ Furthermore, ET/PEP can efficiently identify abnormal LV-aorta coupling in stable patients with HF. ${ }^{49}$ Thus, this measurement device could efficiently support the evaluation or prediction of a decline in LV function and HF. Furthermore, it has been reported that baPWV ${ }^{50-53}$ and aortic stiffness ${ }^{54,55}$ are independent predictors of the onset of hypertension. With regard to the Japanese population only, a cutoff value of approximately $14 \mathrm{~m} / \mathrm{s}$ was shown to significantly raise the OR and the hazard ratio. ${ }^{50-52}$ One reason for the baPWV cut-off value at $14 \mathrm{~m} / \mathrm{s}$ is that this represents "middle-risk"; this cutoff is recommended for lifestyle modifications in the guidelines for "vascular function tests" published by the Japanese Circulation Society. 1,51 Therefore, it is implied that baPWV is able to stratify normotensive patients at higher risk of the onset of hypertension. Needless to say, genetic factors are also considered as one of the causes of hypertension. ${ }^{2}$ Yet, the mechanism involved is not understood because the onset of hypertension is also strongly affected by a combination of acquired and environmental factors. ${ }^{2}$ Thus, to predict the onset of hypertension, the use of baPWV testing is both simple and economical, ${ }^{1,5}$ and could be a more realistic measure than those available at present. Once hypertension develops, a natural consequence is for arterial stiffness to progress synergistically. The extent of the accumulating damage upon the vessels would depend upon the duration for which hypertension was untreated and the internal pressure of the arteries. As a result, even if hypertension is controlled for a certain period after its onset, it follows that the baPWV of such patients gets higher than it does in patients whose BP is maintained at normal levels. ${ }^{56}$ The baPWV also shows individual differences, even in the pediatric population..$^{21,57-60}$ Tomiyama et al described reference values for baPWV in healthy Japanese adults without atherosclerotic risk factors. ${ }^{21}$ The explanatory power of the relevant quadratic regression curve was limited (male $\mathrm{R}^{2}=0.16$, female $\mathrm{R}^{2}=0.37$, both $p<0.01$ ). This indicates that there are clear individual differences in baPWV, even within populations without any atherosclerotic risk factors. Taking this information together, the wider application of a simple baPWV measurement could be a very practical method over the next few decades. Because this measurement can stratify the high risks of hypertension and HF in Japan. Furthermore, baPWV is an independent predictor for all-cause mortality and cardiovascular events. ${ }^{1,2,8-10}$ We should also consider that, even in young normotensive subjects, if baPWV is higher than the normal range $(>$ mean $+\mathrm{SD})$ according to their ages, then there is a higher risk of developing hypertension earlier. In addition, the subject should receive advice pertaining to lifestyle modifications.

\section{Limitations}

There are several limitations of this study that should be considered when interpreting our conclusions. First, our study design was retrospective and cross-sectional in design, although there was an element of longitudinal design. As such, our results do not prove causation. Nevertheless, we can consider that one causative factor is the so-called "vicious cycle" in that a delay, or the neglect of hypertensive treatment, could exacerbate aortic stiffness, and that progressive aortic stiffness itself would further exacerbate hypertension. Second, we did not consider any information relating to blood samples and medication. We must consider that it is likely that there would be residual confounding factors present in class effects, the quantity of prescribed medications, and also in the combination of medications. Third, we did not consider renal function or estimated glomerular filtration rate (eGFR). It is not the case that chronic kidney disease, or end-stage renal failure alone can predict a poorer prognosis. ${ }^{2,61}$ Nevertheless, recent Mendelian randomization studies have, at least, cast doubt on the causative relationship between cardiovascular prognosis and eGFR or cystatin C. Specifically, causative 
factors related to eGFR and $\mathrm{CAD}^{62}$ and on the association between serum cystatin $C$ and cardiovascular events ${ }^{63}$ are evaluated. In other words, these studies potentially suggest that the surrogate indices of renal function, such as eGFR and serum cystatin $\mathrm{C}$, are only mediating factors of prognosis. Further, those indices have no causality with regard to atherosclerotic events. ${ }^{62,63}$ Therefore, it might be inappropriate to undertake statistical analysis upon eGFR and cystatin $\mathrm{C}$ as independent determinants for baPWV, because we hypothesize that baPWV is a prognostic predictor. Fourth, we excluded some patients as possible ASO patients using not only borderline ABI $(\leq 0.99)$ but also by using high \%MAP $(\geq 45)$ and UT $(\geq 180){ }^{1,11,13}$ Thus, there is a possibility that we may have excluded pseudo-positive ASO. On the other hand, there was a high correlation between \%MAP and UT $(\mathrm{R}>0.8, p<0.00001)$. Thus, even if ABI is normal $(\geq 1.0)$, ASO is likely, especially in those with UT and \%MAP close to the exclusion criteria (i.e. $40<\% \mathrm{MAP}<45$ and $170<\mathrm{UT}<180$ ). Therefore, although we used ASO exclusion criteria in our investigation, the possibility of underestimating baPWV is undeniable, due to pseudo-negative ASO. Fifth, our study design did not include any indices related to home BP. It was impossible to consider relationships relating to masked hypertension, morning BP, nocturnal BP, or obstructive sleep apnea. The current Japanese guidelines for hypertension prioritize home BP rather than clinic BP. ${ }^{2}$ Nevertheless, we analyzed only patients whose clinic systolic BP was $123 \pm 9$ (103-139) mmHg, including hypertensives, after attending our clinic on at least three occasions. It would be reasonable to suppose that antihypertensive treatment was administered in hypertensives at least several days before we measured baPWV. It is also appropriate to consider that H-baPWV arises from the influence of the hypertensive state itself. Sixth, we did not measure aortic calcification; therefore, this influence may have been masked in our analysis. ${ }^{64}$ Finally, our analysis only involved outpatients attending a clinic located in a suburban area of a provincial city in Japan, and featured a relatively small number of subjects. A prospective study on a larger scale is now required to confirm our findings.

\section{Conclusion}

We demonstrated that the hypertensive state itself is an independent determinant of high baPWV in outpatients with atherosclerotic risk factors and in whom systolic BP is controlled to $<140 \mathrm{mmHg}$. These results suggest the importance of treating hypertension from an early stage to control potential effects upon arterial stiffness.

\section{Disclosure}

Dai Ato wrote this article as an academic activity based on the guaranteed right of freedom in academy for the Japanese (Article 23), and on the supreme law provided in Article 98 of the Constitution of Japan. Dai Ato is a former employee of Fukuda Colin (formerly Omron Colin, Nippon Colin) Co., Ltd. Fukuda Colin is one of the distributors of the PWV/ABI device (BP-203RPE series). and reports. Dai Ato reports no other conflicts of interest in this work. Toshitami Sawayama reports no conflicts of interest in this work.

\section{References}

1. Yamashina A, Kario K, Kohara K, et al. [Guidelines for noninvasive vascular function test] (JCS2013). Available from: http://www.j-circ. or.jp/guideline/pdf/JCS2013_yamashina_h.pdf. Accessed September 20, 2017. Japanese.

2. Shimamoto K, Ando K, Fujita T, et al; Japanese Society of Hypertension Committee for Guidelines for the Management of Hypertension. The Japanese Society of Hypertension Committee for Guidelines for the Management of Hypertension. Hypertens Res. 2014;37(4):253-390.

3. Vlachopoulos C, Aznaouridis K, Stefanadis C. Prediction of cardiovascular events and all-cause mortality with arterial stiffness: a systematic review and meta-analysis. J Am Coll Cardiol. 2010;55(13):1318-1327.

4. Ben-Shlomo Y, Spears M, Boustred C, et al. Aortic pulse wave velocity improves cardiovascular event prediction: an individual participant meta-analysis of prospective observational data from 17,635 subjects. J Am Coll Cardiol. 2014;63(7):636-646.

5. Yamashina A, Tomiyama H, Takeda K, et al. Validity, reproducibility, and clinical significance of noninvasive brachial-ankle pulse wave velocity measurement. Hypertens Res. 2002;25(3):359-364.

6. Sugawara J, Hayashi K, Tanaka H. Arterial path length estimation on brachial-ankle pulse wave velocity: validity of height-based formulas. J Hypertens. 2014;32(4):881-889.

7. Sugawara J, Tanaka H. Brachial-ankle pulse wave velocity: myths, misconceptions, and realities. Pulse (Basel). 2015;3(2):106-113.

8. Vlachopoulos C, Aznaouridis K, Terentes-Printzios D, Ioakeimidis N, Stefanadis C. Prediction of cardiovascular events and all-cause mortality with brachial-ankle elasticity index: a systematic review and meta-analysis. Hypertension. 2012;60(2):556-562.

9. Ohkuma T, Ninomiya T, Tomiyama H; Collaborative Group for J-BAVEL (Japan Brachial-Ankle Pulse Wave Velocity Individual Participant Data Meta-Analysis of Prospective Studies). Brachialankle pulse wave velocity and the risk prediction of cardiovascular disease: an individual participant data meta-analysis. Hypertension. 2017;69(6):1045-1052.

10. Ato D, Takami T. Brachial-ankle pulse wave velocity, mortality, and cardiovascular events. J Cardiovasc Disord. 2015;2(1):1009.

11. Hashimoto T, Ichihashi S, Iwakoshi S, Kichikawa K. Combination of pulse volume recording (PVR) parameters and ankle-brachial index (ABI) improves diagnostic accuracy for peripheral arterial disease compared with ABI alone. Hypertens Res. 2016;39(6):430-434.

12. Yokoyama H, Shoji T, Kimoto E, et al. Pulse wave velocity in lowerlimb arteries among diabetic patients with peripheral arterial disease. J Atheroscler Thromb. 2003;10(4):253-258.

13. Sawayama T. [Up-stroke Time Prolongation as Potential Early Diagnostic Marker of Peripheral Artery Disease - A Longitudinal Study] Japanese J Clin Physiol. 2016;46(2):79-85. [Article in Japanese].

14. Sawayama T, Ochiai M, Marumoto S, Matsuura T, Niki I. Influence of amyl nitrite inhalation on the systolic time intervals in normal subjects and in patients with ischemic heart disease. Circulation. 1969;40(3):327-335. 
15. Shimizu M, Fujii H, Yamawake N, Nishizaki M. [Prognostic Value of Systolic Time Interval Analysis on Pulse Wave Examination for Poor Left Ventricular Contraction and Dyssynchrony] Japanese J Clin Physiol. 2016;46(2):87-94. [Article in Japanese]

16. The Japan Diabetes Society. Evidence-based Practice Guideline for the Treatment for Diabetes in Japan 2013. Available from: http://www.jds. or.jp/modules/en/index.php?content_id=44. Accessed September 20, 2017.

17. Japan Atherosclerosis Society. Japan Atherosclerosis Society (JAS) Guidelines for Prevention of Atherosclerotic Cardiovascular Diseases 2012 (June 20, 2012). Available from: http://www.j-athero.org/en/ publications/guideline2012.html. Accessed September 20, 2017.

18. Kanda Y. Investigation of the freely available easy-to-use software "EZR" for medical statistics. Bone Marrow Transplant. 2013;48(3):452-458.

19. Xu M, Huang Y, Xie L, et al. Diabetes and risk of arterial stiffness: a mendelian randomization analysis. Diabetes. 2016;65(6):1731-1740.

20. Yao WM, Zhang HF, Zhu ZY, et al. Genetically elevated levels of circulating triglycerides and brachial-ankle pulse wave velocity in a Chinese population. J Hum Hypertens. 2013;27(4):265-270.

21. Tomiyama H, Yamashina A, Arai T, et al. Influences of age and gender on results of noninvasive brachial-ankle pulse wave velocity measurement-a survey of 12517 subjects. Atherosclerosis. 2003;166(2):303-309.

22. Liu Z, Burgess S, Wang Z, et al. Associations of triglyceride levels with longevity and frailty: a Mendelian randomization analysis. Sci Rep. 2017;7:41579.

23. Ichihara A, Hayashi M, Koura Y, Tada Y, Kaneshiro Y, Saruta T. Longterm effects of statins on arterial pressure and stiffness of hypertensives. J Hum Hypertens. 2005;19(2):103-109.

24. Hongo M, Kumazaki S, Izawa A, et al. Low-dose rosuvastatin improves arterial stiffness in high-risk Japanese patients with dyslipidemia in a primary prevention group. Circ J. 2011;75(11):2660-2667.

25. Toyama K, Sugiyama S, Oka H, et al. Combination treatment of rosuvastatin or atorvastatin, with regular exercise improves arterial wall stiffness in patients with coronary artery disease. PLoS One. 2012;7(7):e41369.

26. Ochi M, Kohara K, Tabara Y, et al. Arterial stiffness is associated with low thigh muscle mass in middle-aged to elderly men. Atherosclerosis. 2010;212(1):327-332.

27. Tamakoshi A, Yatsuya H, Lin Y; JACC Study Group. BMI and all-cause mortality among Japanese older adults: findings from the Japan collaborative cohort study. Obesity (Silver Spring). 2010;18(2):362-369.

28. Berrington de Gonzalez A, Hartge P, Cerhan JR, et al. Body-mass index and mortality among 1.46 million white adults. $N$ Engl J Med. 2010;363(23):2211-2219.

29. Tobias DK, Pan A, Jackson CL, et al. Body-mass index and mortality among adults with incident type 2 diabetes. $N$ Engl $\mathrm{J} \mathrm{Med}$. 2014;370(3):233-244.

30. Flegal KM, Kit BK, Orpana H, Graubard BI. Association of all-cause mortality with overweight and obesity using standard body mass index categories: a systematic review and meta-analysis. JAMA. 2013;309(1):71-82.

31. Choo J, Shin C, Barinas-Mitchell E, et al. Regional pulse wave velocities and their cardiovascular risk factors among healthy middle-aged men: a cross-sectional population-based study. BMC Cardiovasc Disord. 2014;14:5.

32. Kim JW, Park CG, Hong SJ, et al. Acute and chronic effects of cigarette smoking on arterial stiffness. Blood Press. 2005;14(2):80-85.

33. Tomiyama $\mathrm{H}$, Hashimoto $\mathrm{H}$, Tanaka $\mathrm{H}$, et al. Continuous smoking and progression of arterial stiffening: a prospective study. J Am Coll Cardiol. 2010;55(18):1979-1987.

34. Kubozono T, Miyata M, Ueyama K, et al. Acute and chronic effects of smoking on arterial stiffness. Circ J. 2011;75(3):698-702.

35. Fukui T, Maruyama M, Yamauchi K, Abe Y, Yoshitaka S, Yasuda T. Ten-year longitudinal study on brachial-ankle pulse wave velocity (baPWV) in middle-aged Japanese males - analysis of relationship with clustering of atherosclerosis risk factors. Ningen Dock International. 2015;2:70-75.
36. Park W, Miyachi M, Tanaka H. Does aerobic exercise mitigate the effects of cigarette smoking on arterial stiffness? J Clin Hypertens (Greenwich). 2014;16(9):640-644.

37. Ji Y, Ge J, Zhu Z, Wang F, Jiang J, Cao H. Relationship between C242T polymorphism and arterial stiffness in an apparently healthy population. J Hum Hypertens. 2016;30(8):488-492.

38. Su HM, Lee KT, Chu CS, et al. Effects of heart rate on brachial-ankle pulse wave velocity and ankle-brachial pressure index in patients without significant organic heart disease. Angiology. 2007;58(1):67-74.

39. Sonoda H, Takase H, Dohi Y, Kimura G. Factors associated with brachial-ankle pulse wave velocity in the general population. J Hum Hypertens. 2012;26(12):701-705.

40. Sugawara J, Komine H, Hayashi K, et al. Effect of systemic nitric oxide synthase inhibition on arterial stiffness in humans. Hypertens Res. 2007;30(5):411-415.

41. Swierblewska E, Hering D, Kara T, et al. An independent relationship between muscle sympathetic nerve activity and pulse wave velocity in normal humans. J Hypertens. 2010;28(5):979-984.

42. Tomiyama H, Hashimoto $\mathrm{H}$, Tanaka $\mathrm{H}$, et al; baPWV/cfPWV Collaboration Group. Synergistic relationship between changes in the pulse wave velocity and changes in the heart rate in middle-aged Japanese adults: a prospective study. J Hypertens. 2010;28(4):687-694.

43. Shimokawa H, Miura M, Nochioka K, Sakata Y. Heart failure as a general pandemic in Asia. Eur J Heart Fail. 2015;17(9):884-892.

44. Konishi M, Ishida J, Springer J, et al. Heart failure epidemiology and novel treatments in Japan: facts and numbers. ESC Heart Fail. 2016;3(3):145-151.

45. Meguro T, Nagatomo Y, Nagae A, et al. Elevated arterial stiffness evaluated by brachial-ankle pulse wave velocity is deleterious for the prognosis of patients with heart failure. Circ J. 2009;73(4):673-680.

46. Aisu H, Saito M, Inaba S, et al. Association of worsening arterial stiffness with incident heart failure in asymptomatic patients with cardiovascular risk factors. Hypertens Res. 2017;40(2):173-180.

47. Cong T, Jiang S, Wang K, Zhong L, Wu J, Su D. Predictive value of brachial-ankle artery pulse wave velocity to heart failure with preserved ejection fraction in hospitalised patients with acute dyspnoea. Pak $J$ Med Sci. 2015;31(3):516-521.

48. Chen SC, Chang JM, Liu WC, et al. Significant correlation between ratio of brachial pre-ejection period to ejection time and left ventricular ejection fraction and mass index in patients with chronic kidney disease. Nephrol Dial Transplant. 2011;26(6):1895-1902.

49. Cheng HM, Yu WC, Sung SH, Wang KL, Chuang SY, Chen CH. Usefulness of systolic time intervals in the identification of abnormal ventriculo-arterial coupling in stable heart failure patients. Eur J Heart Fail. 2008;10(12):1192-1200.

50. Takase H, Dohi Y, Toriyama T, et al. Brachial-ankle pulse wave velocity predicts increase in blood pressure and onset of hypertension. $\mathrm{Am}$ J Hypertens. 2011;24(6):667-673.

51. Tomiyama H, Matsumoto C, Yamada J, et al. Predictors of progression from prehypertension to hypertension in Japanese men. Am J Hypertens. 2009;22(6):630-636.

52. Satoh H, Saijo Y, Kishi R, Tsutsui H. Brachial-ankle pulse wave velocity is an independent predictor of incident hypertension in Japanese normotensive male subjects. Environ Health Prev Med. 2011;16(4):217-223.

53. Zheng X, Jin C, Liu Y, et al. Arterial stiffness as a predictor of clinical hypertension. J Clin Hypertens (Greenwich). 2015;17(8):582-591.

54. Liao D, Arnett DK, Tyroler HA, et al. Arterial stiffness and the development of hypertension. The ARIC study. Hypertension. 1999;34(2):201-206.

55. Najjar SS, Scuteri A, Shetty V, et al. Pulse wave velocity is an independent predictor of the longitudinal increase in systolic blood pressure and of incident hypertension in the Baltimore Longitudinal Study of Aging. J Am Coll Cardiol. 2008;51(14):1377-1383.

56. Doba N, Tokuda Y, Tomiyama H, Goldstein NE, Kushiro T, Hinohara S. Changes in ankle brachial pulse wave velocity during a five-year follow-up period in older Japanese adults: sub-analysis results of the health research volunteer study in Japan. Intern Med. 2013;52(1):21-27. 
57. Niboshi A, Hamaoka K, Sakata K, Inoue F. Characteristics of brachial-ankle pulse wave velocity in Japanese children. Eur J Pediatr. 2006;165(9):625-629.

58. Shiotani A, Motoyama M, Matsuda T, Miyanishi T. Brachial-ankle pulse wave velocity in Japanese university students. Intern Med. 2005;44(7):696-701.

59. Watanabe Y, Masaki H, Yunoki Y, et al. Ankle-brachial index, toebrachial index, and pulse volume recording in healthy young adults. Ann Vasc Dis. 2015;8(3):227-235.

60. Li S, Chen W, Srinivasan SR, Berenson GS. Childhood blood pressure as a predictor of arterial stiffness in young adults: the Bogalusa heart study. Hypertension. 2004;43(3):541-546.
61. Nihon Jinzo Gakkai. [Special issue: Evidence-based practice guideline for the treatment of CKD]. Nihon Jinzo Gakkai Shi. 2013;55(5): 585-860. [Article in Japanese]

62. Charoen P, Nitsch D, Engmann J, et al; UCLEB Consortium. Mendelian Randomisation study of the influence of eGFR on coronary heart disease. Sci Rep. 2016;6:28514.

63. van der Laan SW, Fall T, Soumaré A, et al. Cystatin C and cardiovascular disease: a Mendelian randomization study. J Am Coll Cardiol. 2016;68(9):934-945.

64. Nakamura U, Iwase M, Nohara S, Kanai H, Ichikawa K, Iida M. Usefulness of brachial-ankle pulse wave velocity measurement: correlation with abdominal aortic calcification. Hypertens Res. 2003;26(2):163-167.

\section{Publish your work in this journal}

Vascular Health and Risk Management is an international, peerreviewed journal of therapeutics and risk management, focusing on concise rapid reporting of clinical studies on the processes involved in the maintenance of vascular health; the monitoring, prevention and treatment of vascular disease and its sequelae; and the involvement of metabolic disorders, particularly diabetes. This journal is indexed on PubMed Central and MedLine. The manuscript management system is completely online and includes a very quick and fair peer-review system, which is all easy to use. Visit http://www.dovepress.com/ testimonials.php to read real quotes from published authors. 